DOI: $10.30972 /$ eitt.704767

\title{
Desempeño de productos: aspectos tecnológicos más relevantes de los dispositivos y luminarias LEDs
}

\author{
E. F. Scozzina*, V.Marder, J.L.Ramírez José L. Fontana, \& A. de J. Lin
}

\section{Resumen}

El objetivo de esta publicación es presentar algunos aspectos de la tecnología que sustenta el uso actual de las lámparas LEDs. El trabajo se realiza dentro del Proyecto de Investigación P19Foog de Ciencia y Técnica UNNE.

Como consumidores hemos observado que, en los últimos años, la tecnología LEDs de iluminación ha experimento un gran avance, y ha entrado en nuestras vidas como una alternativa a las lámparas tradicionales, aportado eficiencia y comodidad. El negocio mundial de estas lámparas y luminarias genera millones de dólares al año y se encuentra en franco crecimiento, sin lugar a dudas, resulta ser un rubro, donde se han hecho innumerables innovaciones en materiales y componentes electrotécnicos, con el objetivo de lograr desarrollos competitivos y eficientes, que son requeridos por el mercado.

\section{Introducción.}

Como antecedentes históricos recordemos que las primeras observaciones del fenómeno de electroluminiscencia fueron hechas por Henry Joseph Round en 1907, en los laboratorios Marconi, (Inglaterra). El experimento de Round, originalmente 
utilizó un contacto tipo "bigote de gato" aplicado sobre un cristal CSi (Carburo de Silicio), para observar el fenómeno de las corrientes asimétricas en ese material, descubriendo sin querer, la propiedad electroluminicente del material. Más tarde, en 1927 Oleg Vladimirovich Lósev, realizo la primer publicación detallada sobre la emisión de luz de materiales inorgánicos al aplicarles una corriente eléctrica, " $\mathrm{Lu}$ minous Carborundum Detector and Detection Effect and Oscillations with Crystals”. Algunos años más tarde en 1962, en los laboratorios de General Electric, Nick Holonyak inventó el primer dispositivo LED de emisión en el espectro visible. El desarrollo clave, llego de la mano de Shuji Nakamura, quien es considerado el inventor el primer LED GaN de alto brillo (Nitruro de Galio), fabricó su prototipo en 1993 y ese modelo con distintas mejoras, es el que básicamente se utiliza comercialmente desde el año 1997.

\section{Aspectos normativos legales.}

Como antecedentes legales en la República Argentina, podemos citar la Resolución No 795/2019 Secretaria de Comercio Interior del MPyT, las Norma IRAM 62404-3 y Norma ISO/IEC 17067 y la Ley No 24.240, un hito importante fue el Decreto $\mathrm{N}^{\circ}$ 140/2007 que declaró de interés y prioridad nacional el uso racional y eficiente de la energía, además de ello fijo los lineamientos PRONUREE.

Es contundente la Resolución No 319/99 SICyM del MEyOSP que establece el cumplimiento de las normas para fabricantes, importadores, distribuidores y comerciantes de aparatos eléctricos, esto incluye a los equipos de iluminación y sus accesorios. La resolución establece la obligación del cumplimiento de las Normas IRAM relativas a la eficiencia energética, además de que el producto debe contar con una etiqueta, donde se informa al consumidor sobre este parámetro y otras características técnicas asociadas. Como resguardo para el consumidor, el Art $4^{\circ}$ de la Ley $\mathrm{N}^{\mathrm{O}}$ 24.240, establece que los fabricantes nacionales e importadores tienen responsabilidad técnica, civil y penal relacionada a los productos, por la fabricación, importación o comercialización.

En los requerimientos técnicos y características esenciales necesarias para el etiquetado, que deben cumplir las lámparas LEDs, estas deben exhibir sobre la caja o 
embalaje la etiqueta de Eficiencia Energética, cuyas características se detallan en la Norma IRAM 62404-3 punto 9, sumado a la vida nominal de la lámpara expresada en horas de uso. Es importante aclarar para el consumidor que el tipo de etiqueta utilizada es del tipo "Etiqueta obligatoria sin estándar mínimo”. El cumplimiento de estas exigencias establecidas se verifica mediante una certificación que es otorgada por un organismo de certificación acreditado, de acuerdo con los lineamientos establecidos en la Norma ISO/IEC 17067 Esquema 5.

Una correcta definición de modelo para una lámpara LED, implica que deberán determinarse, los parámetros eléctricos y luminotécnicos, además de otras características técnicas de la lámpara. Por ejemplo: Grupo de clasificación, Tipo de Casquillo (rosca), Formato del bulbo, Potencia, Temperatura de color en K, Flujo luminoso, Componentes de la lámpara y de su controlador de alimentación drivers. Sabemos de las lámparas LED ahorran dinero, dado que tienen mayor eficiencia energética en relación al flujo luminoso, además un bajo mantenimiento y no se rompen fácilmente, por lo que mejoran la seguridad en lugares de trabajo.

Veamos las ecuaciones que describen su comportamiento general, el rendimiento de una lámpara, compuesta por un conjunto de n LEDs está dado por:

$$
\text { Rendimiento }=\frac{\text { Potencia luminica } n \text { LEDs }}{\text { Perdidas en driver }+ \text { Perdidas en los } n \text { LEDS }}
$$

Para el caso de un dispositivo LED:

$$
P_{\text {electrica }}=V_{F}(T) \times I_{F}(T)=\left(P_{\text {heat }}+\eta P_{\text {electrica }}\right)=\left(P_{\text {heat }}+\Phi_{e}\right)
$$

\begin{tabular}{|c|l|c|}
\hline Potencia eléctrica & Potencia radiante & Disipación de calor \\
\hline$P_{\text {electrica }}=V_{F} \times I_{F}$ & $P_{\text {radiante }}=\Phi_{e}=\eta P_{\text {electrica }}$ & $P_{\text {heat }}=P_{\text {electrica }}-\Phi_{e}$ \\
\hline
\end{tabular}

La Eficacia luminosa típica de la radiación, es expresa por los fabricantes en lúmenes/watt, esta varía según el tipo y modelos de LED, pero como regla general, podemos citar por ejemplo: LEDs blancos ronda $325 \mathrm{~lm} / \mathrm{W}$, amarillos $480 \mathrm{~lm} / \mathrm{W}$, rojos $130 \mathrm{~lm} / \mathrm{W}$, y el ámbar $240 \mathrm{~lm} / \mathrm{W}$. 
Los dispositivos LED tienen una vida útil más larga, su ciclo de vida llega a las decenas de miles de horas, lo que es exponencialmente más largo que otros sistemas de iluminación convencionales. Al superar el número de horas nominal, continúan emitiendo su luz, aunque comienzan a perder brillo y luego se funden.

\section{Consideraciones sobre el color.}

Las lámparas LEDs poseen una buena reproducción cromática, la propiedad luminotécnica asociada, es la temperatura de color, este concepto se utiliza en iluminación general y relaciona con el color de la luz producida por una fuente, con las temperaturas de color en grados Kelvin. Una lámpara incandescente, genera toda una gama de colores en espectro visible entre los 2.700 y $3.500 \mathrm{~K}$, cuanto menor sea el valor de la temperatura, más cálida es la sensación de la luz, (aspecto más amarillo, tendiendo al naranja). Por ejemplo comercialmente contamos con lámparas LEDs blanco cálido están en el rango de los 2.800-3.50oK, un blanco natural 4.oooK y blanco frío supera los 5.00oK. En el caso de los dispositivos LEDs individualmente, para dispositivos de montaje superficial SMD, un fabricante reconocido clasifica la temperatura de color, de la siguiente manera: White (11.00oK-4.60oK), Warm White (4.600K-2.50oK) y Candle Color (2.580K-1.870K), son algunos de los distintos modelos led producidos.
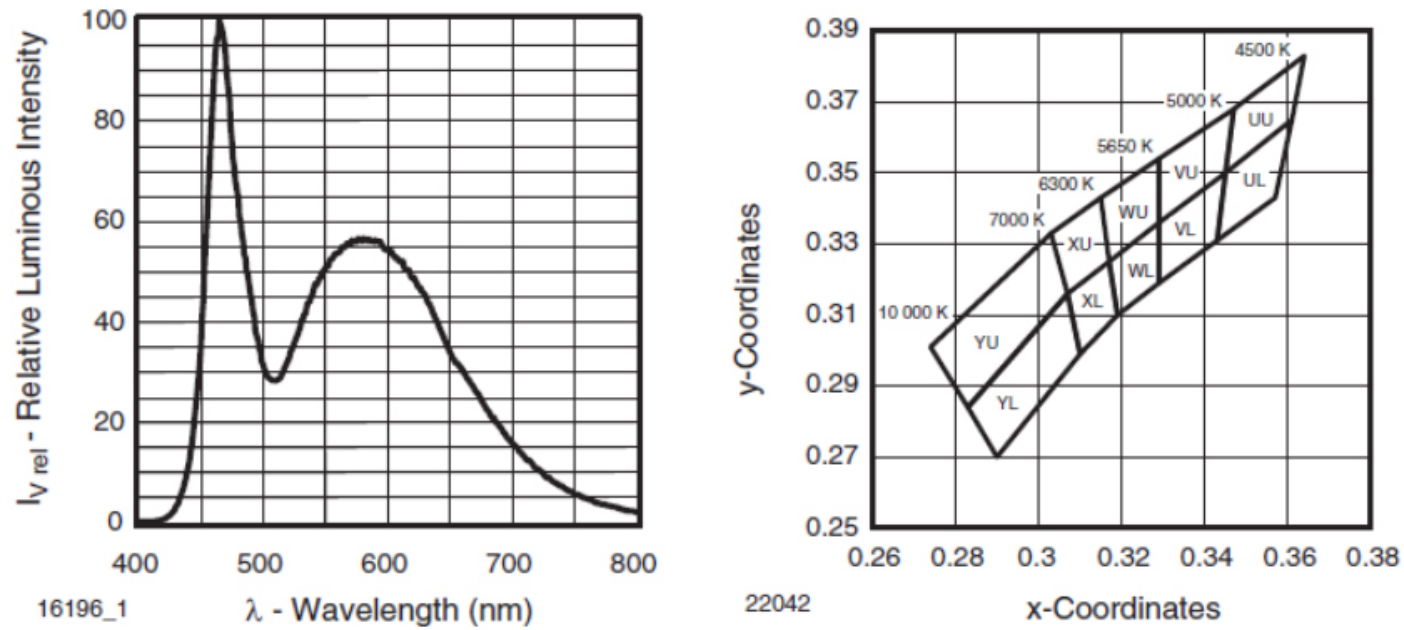

Figura: a la izquierda, se muestra la intensidad relativa en función la distribución de la longitud de onda emitida. A la derecha se muestra en detalle el grupo de coordenadas cromáticas para distintos modelos de LEDs de blancos, proporcionadas por un fabricante. 
Una propiedad muy importante es el mantenimiento del flujo luminoso en una lámpara. Este parámetro debe ser ensayado según la norma IEC 62612:2013. El ensayo típico dura 3.000 horas, las lámparas de muestra deben mantener un flujo determinado, que dependerá de su vida nominal en horas. Para este ensayo, se admite una tolerancia del $3 \%$ al valor de flujo luminoso.

\begin{tabular}{|l|l|}
\hline Vida nominal de la lámpara LEDs en horas & $@ 3,3000$ horas \\
\hline Hasta 10.000 & $83,7 \%$ \\
\hline 10.000 a 15.000 & $89,9 \%$ \\
\hline 15.000 a 20.000 & $93,1 \%$ \\
\hline 20.000 a 25.000 & $94,8 \%$ \\
\hline 25.000 a 30.000 & $95.8 \%$ \\
\hline 30.000 a 40.000 & $96,5 \%$ \\
\hline 40.000 a 50.000 & $97,4 \%$ \\
\hline Mayor a 50.000 & $97,9 \%$ \\
\hline
\end{tabular}

\section{LEDs Driver utilizados.}

Los como dispositivo LEDs trabajan con polarización de corriente continua, por lo cual se debe adecuar la corriente de red eléctrica convencional (220V-50Hz). Se debe contar con un circuito rectificador a diodos que convierte la corriente alterna en corriente continua y luego fíltrala, los modelos más eficientes cuentan con un controlador (driver) que maneja la corriente sobre el conjunto de LEDs, conectados en serie, aunque también existen otros arreglos de tipo serie/paralelo.

En mayoría de las lámparas LED, se utilizan dispositivos SMD, los LEDs están conectados en serie formado un lay out adecuado junto a otros componentes sobre una plaqueta de tipo MCPCB. Se debe contar con un diseño adecuado de compatibilidad electromagnética, para estoo se adiciona un filtro a la entrada, con el objeto de disminuir las emisiones electromagnéticas conducidas, que se puedan propagar sobre la red.

Un driver de buena calidad debe asegurar el funcionamiento estable de lámpara, en el caso de variaciones de tensión en la red. El control mediante drivers de CI resulta más sofisticado y eficiente, comparado con el modelo RC, esto impacta directamente en el precio final de la luminaria y mejora la vida útil. Un circuito integrado sencillo, es el PT 4515C que fija la corriente de los LEDs mediante una resistencia externa, que se ubica en los terminales 3 y 2 y se calcula según la ecuación. 


$$
I_{\text {LED }}=\frac{600 \mathrm{mV}}{R_{\text {Externa }}}[\mathrm{mA}]
$$


Figura Montaje típico de un Driver Led PT 4515 de PowTech utilizado en una lámpara de marca reconocida en nuestro país.

Son numerosos los circuitos drivers y topografías utilizadas como soluciones en lámparas de venta común, y además existe una proliferación fabricantes de circuitos integrados que abordan esta sencilla, pero rentable aplicación, entre los más reconocidos mencionamos: Vishay, Kingbrigth, Nichia, Xlsemi, Joulewatt, además de otras compañías orientales incipientes. Los CI's más comunes que se obtuvieron de un relevamiento hecho sobre lámparas del mercado local son: JW1792, BP2831A, SM7525, CPC9909.

Un dispositivo utilizado por su sencillez y economía, es el regulador lineal de corriente constante (CCR), están montados en un encapsulado muy compacto (SOD 123-CASE 42) y diseñado para proporcionar una solución rentable en la regulación de la corriente en circuitos con múltiples LED`s. El CI es de tecnología (SBT -SelfBiased Transistor), lo que permite regular la corriente en un amplio rango de voltaje de entrada. La Rth juntura carcasa es del orden $360^{\circ} \mathrm{C} / \mathrm{W}$, la cual baja al ser montado en un MCPCB que actúa como disipador y por su pequeño tamaño se manejan corrientes de miliamperios, esta tecnología también permite el desvanecimiento (dimming) mediante el agregado de un transistor en serie adicional, controlado en su base por un circuito PWM.

Por ejemplo CCR NSI4509oJDT4G utilizados, para reemplazos de tubos fluorescentes $\mathrm{T}_{5}$, se conecta de manera directa conectada a la red de CA 220/110 V mediante un puente rectificador y filtro capaccitivo. 
Los circuitos más sencillos denominados a tensión constate ó RC Capacitor Dropper Circuit, Non-Isolated AC/DC LED Driver, este diseño suele limitar la vida útil de la lámpara, dado que no puede absorber las variaciones de tensión de red y produce un desplazamiento de los puntos de trabajo de la recta de carga, sumado a que genera un factor de potencia capacitivo. Se debe prestar atención cuando existen numerosas lámparas conectadas, con esta tecnología.

$$
\mathrm{Z}=\sqrt[2]{R^{2}+X_{C}{ }^{2}} \text {, done } \theta=\operatorname{tg}^{-1} \frac{R}{X_{C}}
$$

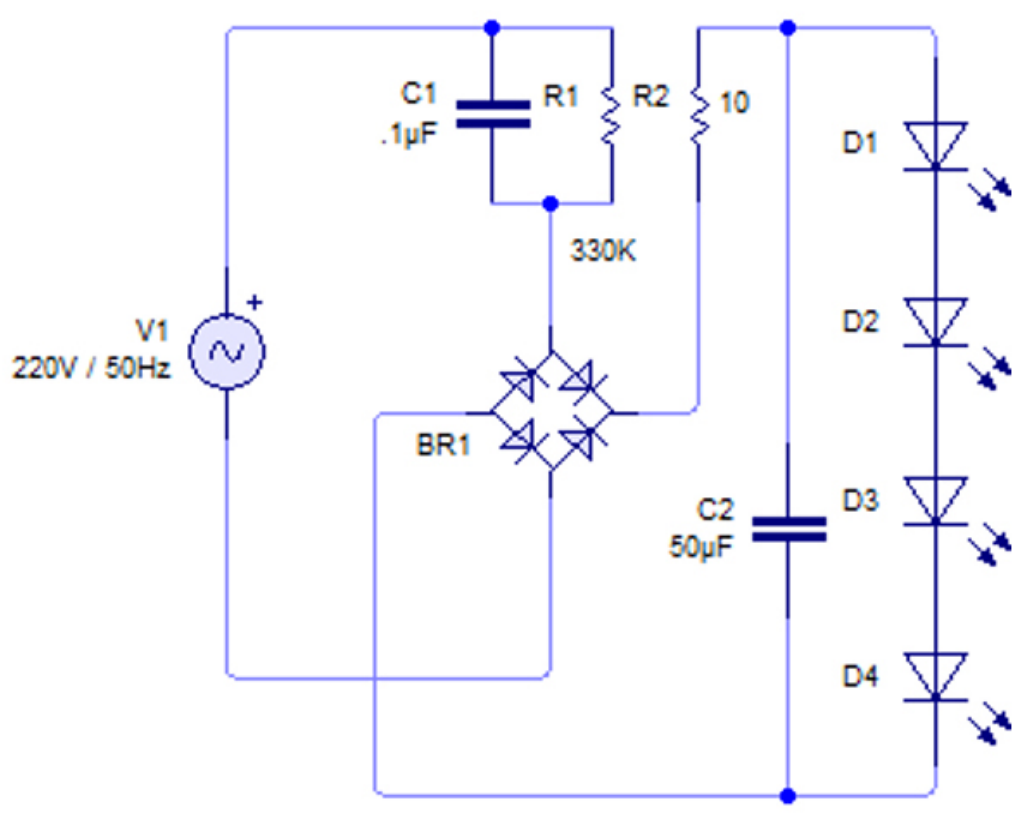

Donde el factor de potencia se expresa como:

$$
\begin{gathered}
\cos \varphi=\frac{P}{S}=F D=\text { factor de desplazamiento } \\
\text { FPotencia }=F D+\text { Factor de distorción armonicos }(F \omega)
\end{gathered}
$$



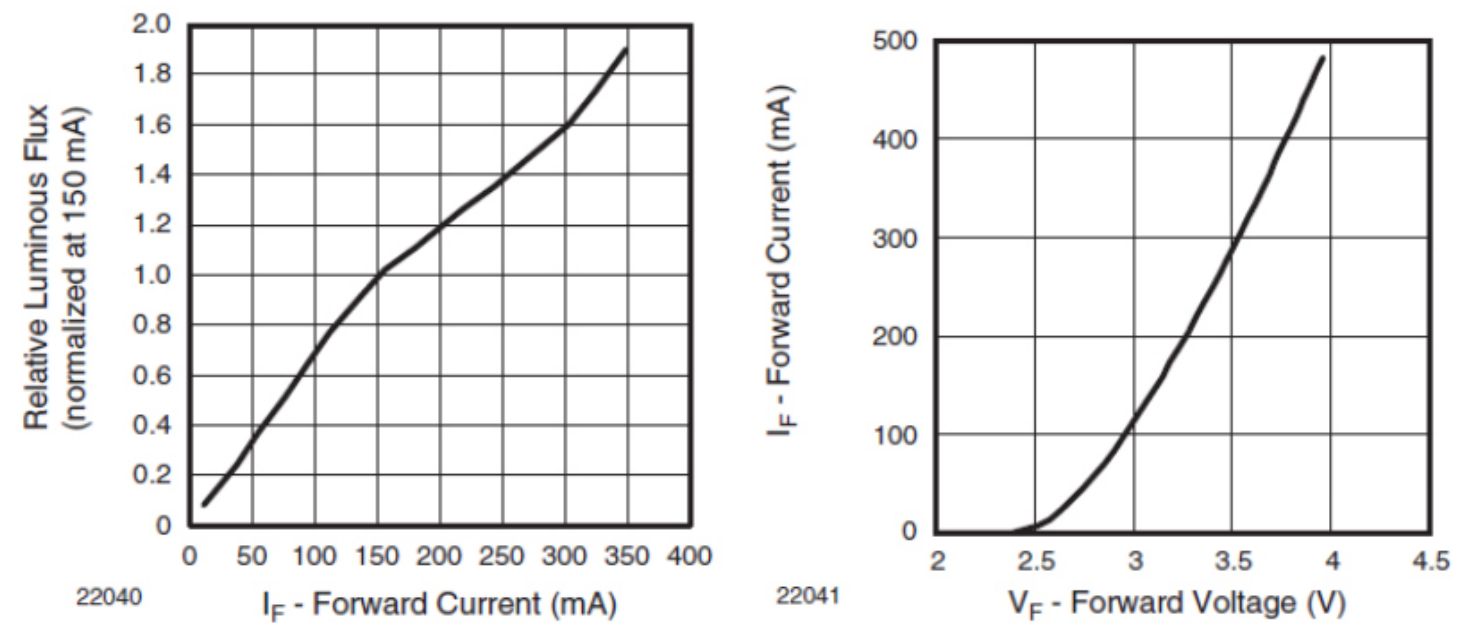

En el gráfico de la izquierda, se muestra la iluminación relativa flujo Luminoso en función de la corriente directa $I_{F}$. En la derecha se muestra la característica tensión-corriente directa $\left(V_{F}\right.$ versus $\left.I_{F}\right)$ para un diodo LED de tecnología InGaN de alta eficiencia. (Vishay).

\section{Gestión térmica de la lámpara.}

Cuando un LEDs está en funcionamiento, disipa de calor por efecto Joule en la unión semiconductora, si la temperatura presenta un excesivo aumento, se produce la fusión térmica de la unión, estropeando el dispositivo o disminuyendo su vida útil.

La fiabilidad del funcionamiento en conjunto, depende de mantener siempre un flujo térmico adecuado, en el caso de luminarias con mayores potencias se utilizan disipadores incorporados, que proporcionan una superficie adicional.

Para caracterizar el comportamiento térmico de un LEDs, debemos analizar la luminaria en conjunto, como un dispositivo genera calor, y comprender por qué medios que se disipa hacia el ambiente, (mecanismo por conducción, en principio y luego por convección). Esto depende del encapsulado y el montaje de soporte utilizado, simplificando se puede modelar mediante tres resistencias térmicas con los siguientes parámetros:

Rthjs $=$ Juntura - Punto de soldadura

Rthsb = Soladura placa MCPCB, depende de la tecnología utilizada.

Rthba = Placa MCPCB -Ambiente 
Tjmax $=$ Temperatura de juntura

Tamb = Temperatura ambiente

$\mathrm{VF}(\mathrm{T})=$ Voltaje directo.

IF $(\mathrm{T})=$ Corriente directa.

$$
P_{L E D}=V_{F} \times I_{F}=\left[\frac{T_{J \max }-T_{a m b}}{\operatorname{Rth}_{J S}+R t h_{S B}+R t h_{B A}}\right]
$$

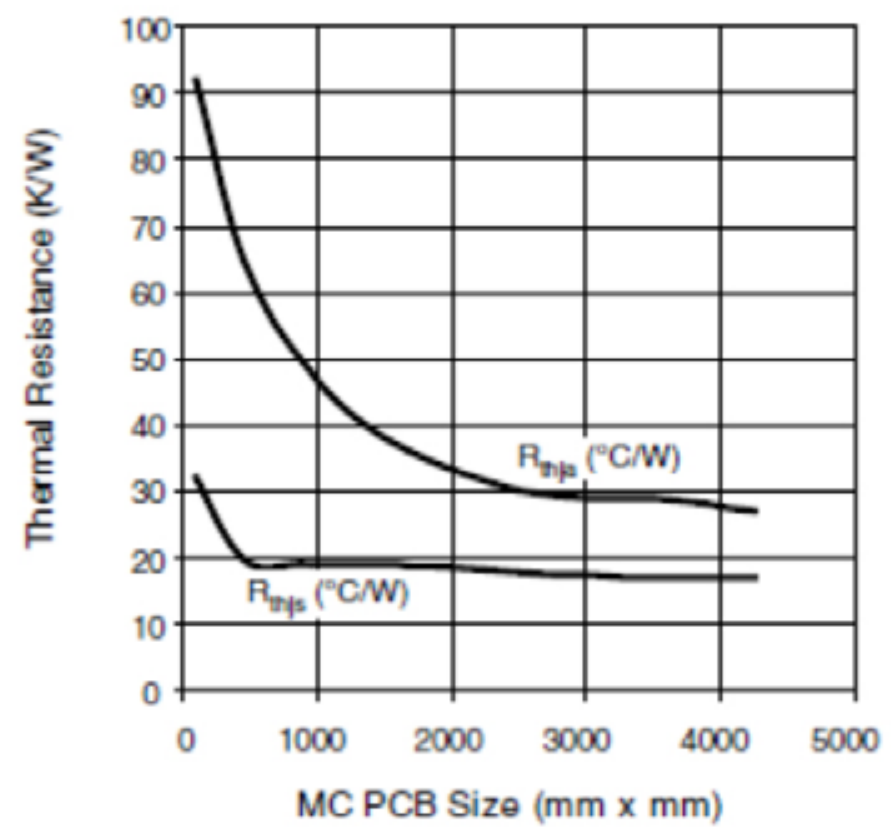

En la figura de la izquierda, sobre el eje de las ordenadas, se puede ver el cambio de la resistencia térmica de la placa MCPCB en función de la superficie de montaje (abscisas).

Una placa MCPCB es una combinación de una placa de metal, con una capa aislante, sumada a las pistas de cobre que proporcionan el camino conductivo al circuito electrónico montado en ella. Al integrar esta ingeniosa solución, nos da como resultado una nueva tecnología con muchos beneficios, si la comparamos con una placa de PCB convencional, en principio por la baja conductividad térmica, no es capaz de hacer frente a las prestaciones de una luminaria. El montaje en un circuito (MCPCB) es una solución que permite evacuar el calor generado por sus componentes individuales de forma controlada, y permite asegurar que la temperatura de los componentes se mantenga dentro de los límites funcionales. 


\section{Fallas en el material.}

Los ensayos de fallas, son utilizados en la industria para determinar la calidad y performance de los materiales y componentes electrotécnicos producidos. En relación a esto existen gran variedad de normas y métodos estadísticos sistematizados para determinar la vida útil de un producto fabricado con semiconductores.

El modelo de Arrhenius, que permite caracterizar el estrés térmico, y describe el tiempo de falla en función de la temperatura en el dispositivo. El mecanismo de fallas, es del tipo no mecánico, muy común en materiales y componentes electrotécnicos. La tasa de falla $\mathrm{F}$ es directamente proporcional a la temperatura y puede ser descrito por la ecuación:

$$
F=A E e^{\frac{E_{A}}{k T}}
$$

Donde $\mathrm{T}$ es la temperatura en grados Kelvin al momento de producirse la falla, $\mathrm{k}$ es la constante de Boltzmann $\left(8,617 \times 10^{-5} \mathrm{ev} / \mathrm{K}\right)$. El valor que toma la constante $\mathrm{A}$, representa un factor de escala y $\Delta \mathrm{H}=\mathrm{E}_{\mathrm{A}}$ es la energía activación en el proceso, dejamos en claro que este último valor es función del tipo de mecanismo de falla y los materiales utilizados, los valores típicos se encuentran entre 0,3 a 1,5.

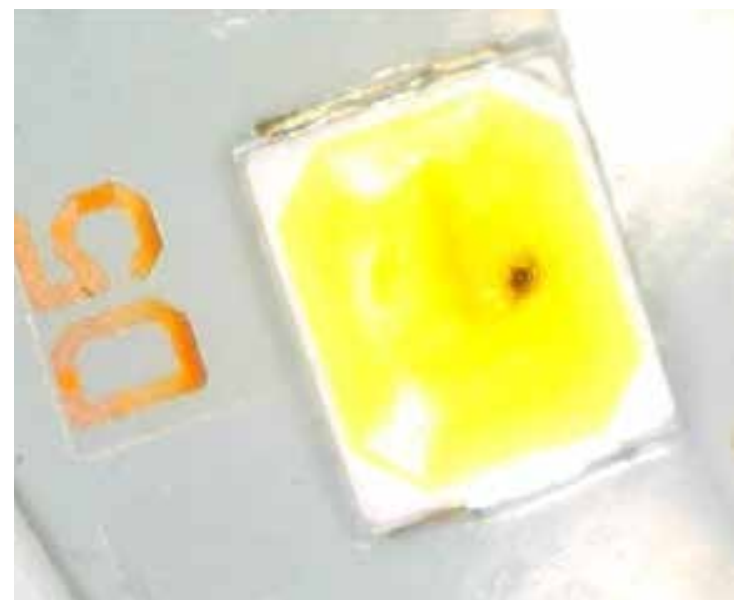

Falla térmica en un diodo led, fotografía del autor. 


\section{Mediciones realizadas sobre lámparas comerciales y de uso general.}

El objetivo fue determinar cómo se comportan las corrientes de lámparas LEDs, para distintas tensiones de alimentación y evaluar su factor de potencia. Para las mediciones se utilizó una lámpara con una resistencia serie, sobre la cual se observó y midió la forma de corriente mediante un osciloscopio digital. Se realizaron distintas determinaciones en lámparas de la misma marca, pero de distinta potencia, 6, 7, 9 y 15 watts.

Mediante un transformador variable conectado a la red, se varió la tensión en subida y bajada, determinado los puntos de encendido y apagado. Se obtuvieron distintos resultados con cada una de las lámparas, tanto en la variación de la corriente, variación del ángulo entre tensión y corriente $(\varphi)$ así como también en la tensión de apagado de las mismas.

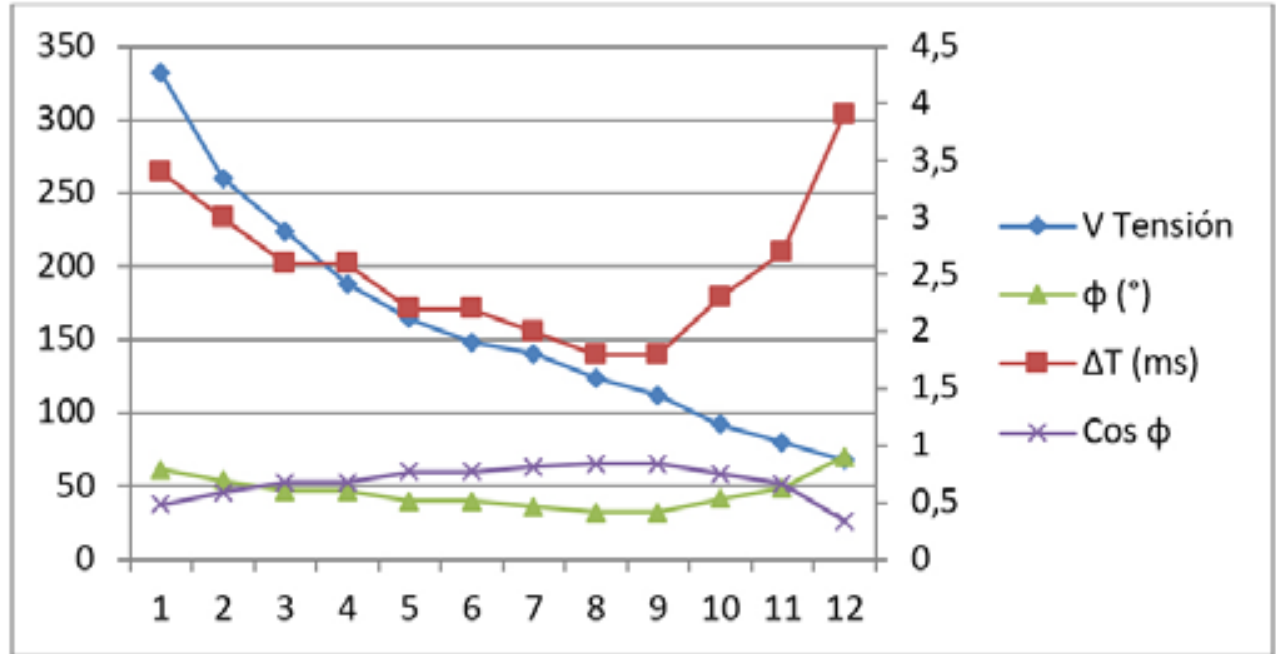

Figura Ejemplos de las series de determinaciones realizadas sobre las lámparas. Resultado de las mediciones realizadas sobre una lámpara led de uso domiciliario de una potencia de $6 \mathrm{~W}$, de marca reconocida. 


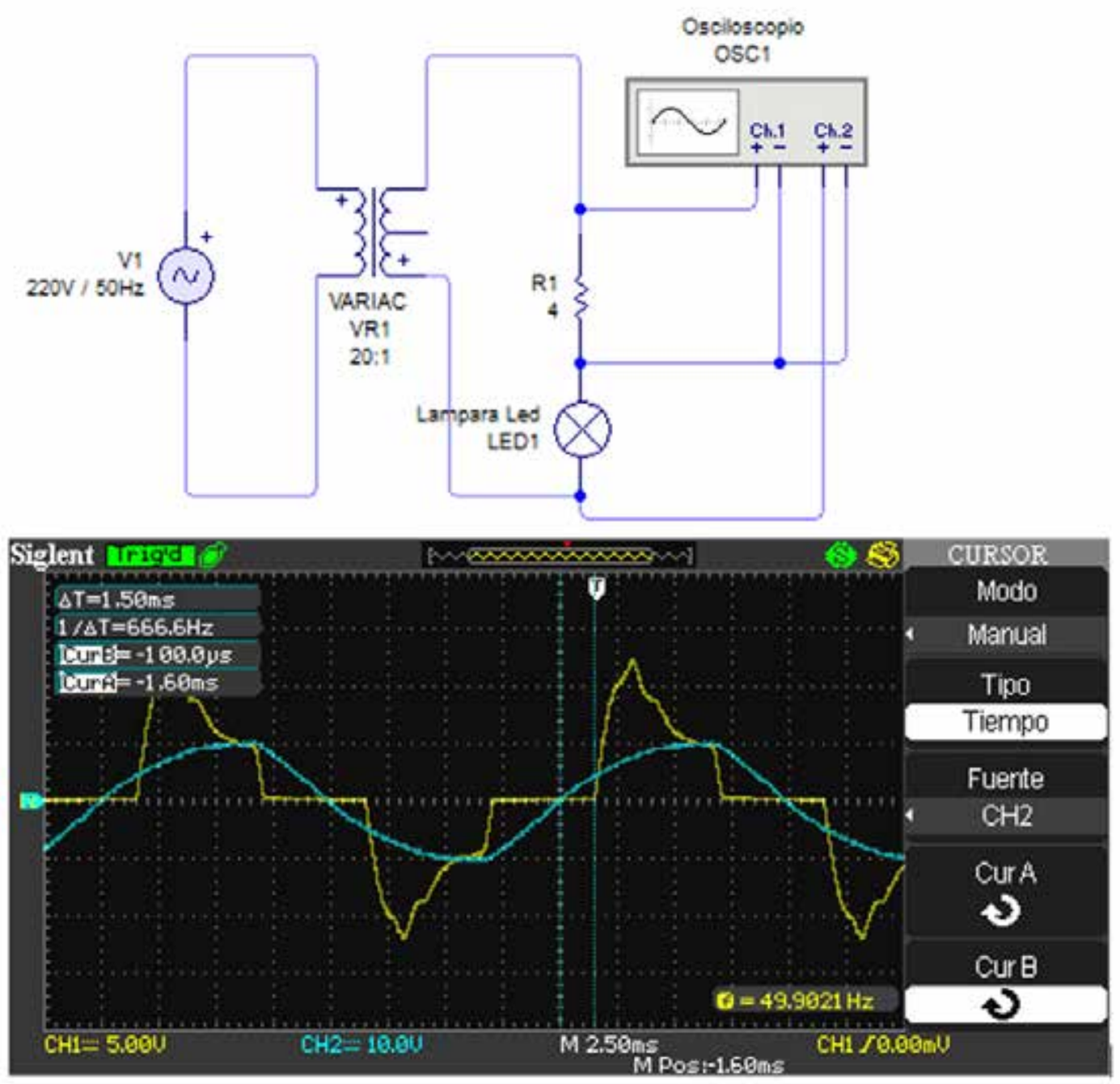

Captura de pantalla del osciloscopio, y circuito de medición utilizado. Se puede ver la forma de la corriente, para este caso.

\section{Conclusiones para el consumidor}

Hasta este punto pareciera que son todas ventajas; Pero ¿cómo consumidores estamos seguros de que un producto LED es los que dice ser? ¿Por ejemplo, una lámpara común cumple con las prestaciones que declara el producto? ¿Quién garantiza que el precio de producto asegure las prestaciones? ¿Qué hacemos con las marcas genéricas o de origen dudoso?

Estas y otras son algunas de las preguntas que nos hacemos, para preservar nuestros derechos como consumidores. En muchas ocasiones nos encontramos con 
diseño electrónico o térmico no adecuado, lo que impacta fundamentalmente en la vida útil del sistema en su conjunto, generando altas tasas de falla y nuevos gastos al reponer las lámparas falladas.

Existen aspectos que saltan a la vista del consumidor, por ejemplo, cuando nota que su lámpara “calienta más de lo normal”, esto sucede, en las manufacturas de baja calidad, donde muchas veces no verifica un manejo térmico adecuado. El uso prolongado de las lámparas requiere un enfoque distinto al problema de disipación térmica mediante la incorporación de una placa de circuito impreso de núcleo metálico, por lo cual esta característica de diseño es muy importante a cumplir.

El uso de lámparas con driver tipo circuitos RC "no compensados”, generan un factor de potencia capacitivo sobre la red eléctrica del usuario, que debe ser subsanado.

En cuanto a ganar nuevas prestaciones, para los usuarios la tecnología de lámparas LEDs, está avanzando hacia las redes de telecomunicaciones con Li-Fi convirtiendo la luz en modo de comunicación para usuarios LiFi es un sistema de transmisión de datos mediante luz visible o VLC (Visible Light Communication). La tecnología puede usar todo el espectro de luz visible, en comparación al WiFi que utiliza usa las bandas de los 2,4 GHz y $5 \mathrm{GHz}$, por lo que todas las conexiones se amontonan en un ancho de banda reducido muchas veces generando problemas de conexión por el uso simultaneo. Las desventajas la tecnología LiFi son dos, 1) para la conexión se requiere la iluminación y 2) se necesitarían muchas lámparas para cubrir una zona.

\section{Bibliografía:}

Round, H. J. (1907), «A note on carborundum», Electrical World 49: 309

NSI45020T1G: Constant Current Regulator \& LED Driver 45 V, 20 mA - 15\%, 460 mW Package. Publication Order Number: NSI45O2O/D Semiconductor Components Industries, LLC, 2014 -June, 2014 - Rev. 6.

Application Note AND8123/D Power Factor Correction Stages Operating in Critical Conduction Mode. Literature Distribution Center for ON Semiconductor $A N D$ 8123/D. 
Application Note No. ANo4O : Comparison of simple LED circuits for low power LEDs. Valid for: visible LEDs; Infrared Emitters. OSRAM.

Resolución 795/2019 de la Secretaría de Comercio Interior y Norma IRAM 62404-3 Resolución No 319/99 SICyM del MEyOSP

Norma ISO/IEC 17067 Evaluación de la conformidad. Fundamentos de la certificación de producto y directrices para los esquemas de certificación de producto.

Ley No 24.240 Normas de Protección y Defensa de los Consumidores.

IEC 62612 Ed. 1.o b: 2013 Self-Ballasted LED Lamps for General Lighting Services with Supply Voltages > $50 \mathrm{~V}$ - Performance Requirements

Donald Fink, Alexander McKenzie, Electronic Engenier`s Handbook Editorial Mc Graw Hill Frist Edition 1975.

Richard C. Dorf, The Electrical Engineerring Handbook. Editorial, CRC Press 1993.

Texas Instruments General lighting LED drivers - Products TPS92411x

Floating Switch for Offline AC Linear Direct Drive of LEDs with Low Ripple Current. Application Note.

LEDs for Lighting Solutions Surface-Mount and Leaded LEDs, Displays, and LED Modules SG2132-20o3. Vishay Semiconductors. Application Note.

Physics of Optoelectronic Devices Vishay Semiconductors. Document - Number: 80o97-Rev.1.2, o5-Jul-04.

Using Metal Core Printed Circuit Board (MCPCB) as a Solution for Thermal Management. by: Winco K.C. Yung, PhD, Associate Professor PCB Technology Centre, Department of Industrial and Systems Engineering, The Hong Kong Polytechnic University

JW1792 -Integrated 50oV MOS Non-isolated Buck LED Driver. JW1792 Rev.o.91 JoulWatt $\mathbb{R}$

http://www.ellwest-pcb.com/metal.php

Application Note O2_o1 TELUX-Specification, Thermal management and design-in-Document Number 81071 Rev. 1.1, o6-Aug.-02. Application Note

Texas Instrumetns: LED drivers: 12oVAC Input 3.5W Output LED Driver Candelabra (E12) \& Small Form Factor LED Ligth Reference Design.

Texas Instrumetns: LED drivers: $230 \mathrm{~V}$ in to $180 \mathrm{~V}$ out at $200 \mathrm{~mA}$ constant-current LED driver reference design PMP30439 